> La membrane plasmique a longtemps été considérée comme une simple barrière biologique séparant le milieu intracellulaire du milieu externe. Cette vision est devenue caduque, car il est maintenant admis que la membrane plasmique joue un rôle prépondérant dans un grand nombre de processus physiologiques puisqu'elle comporte la plupart des éléments essentiels aux échanges entre la cellule et son environnement. D'une part, la membrane plasmique est capable de se structurer pour participer activement à la signalisation intracellulaire nécessaire au maintien de la vie cellulaire; d'autre part, les altérations auxquelles elle est soumise constituent sans doute autant de signaux de transfert intercellulaire d'informations biologiques. Cet article fait le point sur les acquis les plus récents concernant la plasticité membranaire et les mécanismes intracellulaires mis en jeu lors des modifications de la répartition des lipides entre les feuillets de la bicouche membranaire pendant l'activation cellulaire. Les conséquences physiopathologiques de la présence de microparticules/microvésicules résultant de ce remodelage sont brièvement abordées. <

\title{
Remodelage de la membrane plasmique et stimulation cellulaire
}

Maria Carmen Martínez, Corinne Kunzelmann, Jean-Marie Freyssinet

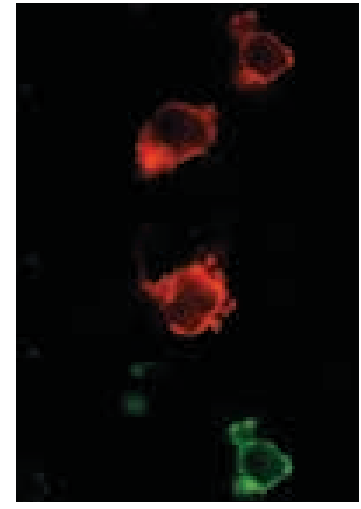

Lors de la perte de cette asymétrie, la surcharge transitoire en PL dans le feuillet externe aboutit à la formation de bourgeons, suivie par l'émission de microparticules (MP). Ces MP sont porteuses de PS, d'antigènes fonctionnels et/ou de molécules d'adhérence de la cellule d'origine. Les mécanismes intracellulaires conduisant à l'externalisation de PS restent à préciser.

La membrane plasmique des cellules eucaryotes est organisée en une bicouche de phospholipides ( $\mathrm{PL}$ ) et de cholestérol dans laquelle sont insérées ou ancrées des protéines. La répartition des PL entre les deux feuillets membranaires est asymétrique: la phosphatidylsérine (PS) est séquestrée du côté cytoplasmique. Le remaniement membranaire entraîne une perte de l'asymétrie et la PS est externalisée. Dans des cellules spécialisées comme les plaquettes, l'externalisation de la PS promeut le déclenchement du processus de coagulation. La PS exposée est un déterminant de l'élimination par phagocytose des cellules apoptotiques ou sénescentes.

\section{Organisation membranaire de la cellule au repos}

La membrane plasmique est composée d'éléments en proportions variables: $50 \%$ de lipides (PL et cholestérol), $45 \%$ de protéines et $5 \%$ de glycanes. Deux classes de PL sont majoritaires: (1) les PL dont la tête polaire est la choline: la phosphatidylcholine (PC) et la sphingomyéline (SM) (neutres au pH physiologique); (2) les aminoPL, PS et phosphatidyléthanolamine $(P E)$, dont la tête polaire est une amine primaire. Au pH physiologique, la PS a une charge nette négative et la $P E$ est globalement neutre. 


\section{Répartition asymétrique des PL}

Dans la bicouche lipidique de la membrane plasmique, les PL sont organisés en phase lamellaire, les acides gras hydrophobes orientés vers l'intérieur(c'est-à-dire disposés en lames ou en lamelles formant une bicouche) et les têtes polaires vers l'extérieur [1]. La répartition des PL entre les deux feuillets de la membrane plasmique est asymétrique (Figure 1) et la PS est retrouvée presque exclusivement dans le feuillet interne.

\section{Domaines membranaires}

Les lipides sont aussi organisés dans le plan latéral. Cette organisation résulte d'un regroupement dynamique préférentiel des sphingolipides et du cholestérol dans des platesformes mobiles ou rafts (radeaux) capables d'inclure ou d'exclure certaines protéines [2]. On y retrouve principalement celles qui sont liées par une ancre glycosylphosphatidylinositol, les protéines doublement acylées, liées au cholestérol, et d'autres protéines transmembranaires, souvent palmitoylées. La distribution des rafts à la surface membranaire varie selon le type et l'état d'activation de la cellule. Pour certains, les rafts ne seraient définis que par les méthodes utilisées pour leur détection ${ }^{1}$. Ainsi, la membrane devient une structure dynamique organisée en domaines transitoires résultant d'interactions «évanescentes» (membranes are a floating world of evanescent associations [3]). L'organisation transverse et latérale de la membrane plasmique lui confère une plasticité permettant son intervention dans toutes les réponses cellulaires.

${ }^{1}$ II existe en effet différentes techniques pour caractériser et isoler les rafts.

\section{Perte de l'asymétrie membranaire lors de l'activation cellulaire: exposition de PS et vésiculation}

Lors de l'activation cellulaire, la répartition asymétrique des PL est rompue et la PS est rapidement exposée [4]. Dès la mise en route du remodelage membranaire, l'influx compensatoire tardif ${ }^{2}$ de $P C$ ou de SM,

${ }^{2}$ La PS est rapidement externalisée à la surface cellulaire, alors que la PC et la SM sont internalisées (influx) plus lentement (tardif) et cela pour compenser l'exposition de la PS (compensatoire)

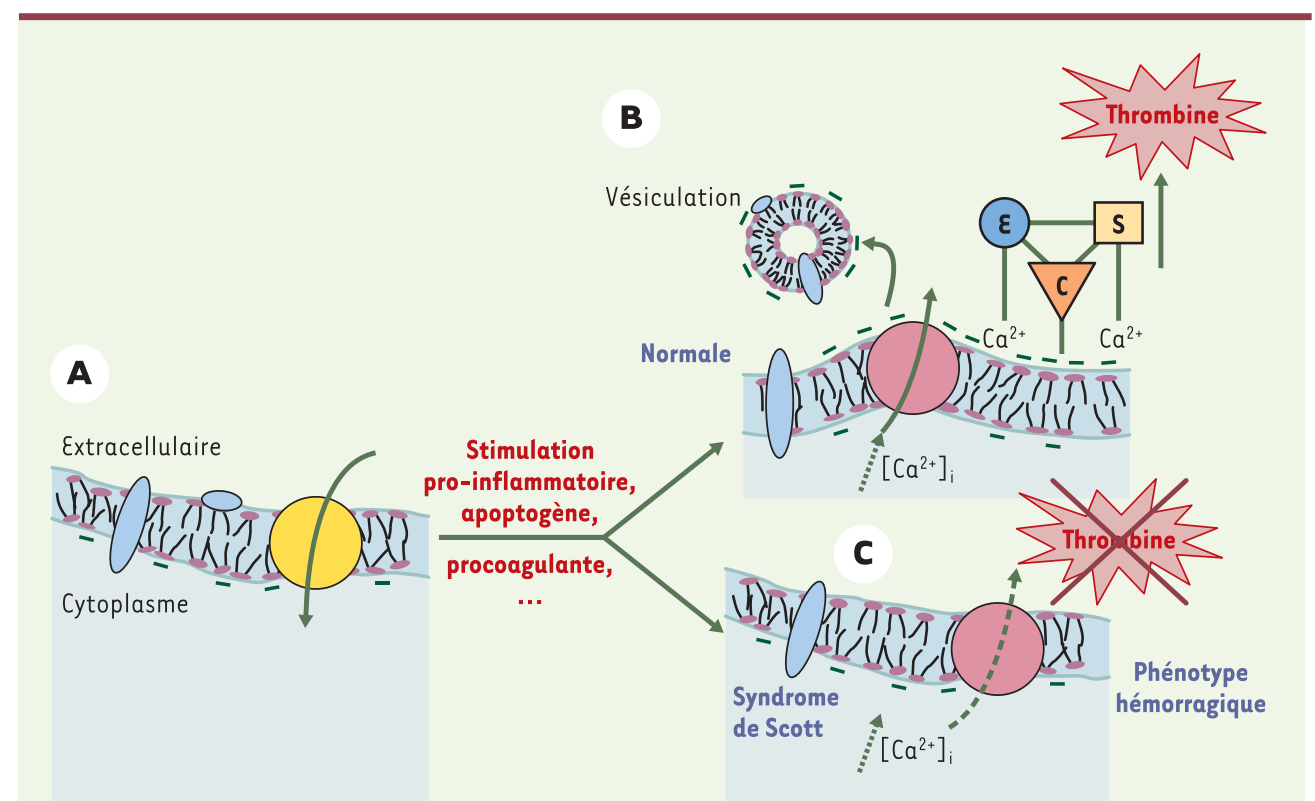

Figure 1. Représentation schématique de la membrane plasmique de la cellule au repos (maintien de l'asymétrie membranaire, A) et de la cellule stimulée (perte de l'asymétrie, B). La phosphatidylsérine (PS) est représentée par sa charge nette négative (signes moins en vert foncé); elle est séquestrée dans le feuillet interne de la cellule au repos sous l'action d'un transporteur, l'aminophospholipide translocase (en jaune). Après stimulation, la concentration intracellulaire de calcium augmente, ce qui déclenche le transport de cet aminophospholipide anionique vers le feuillet externe et provoque une surcharge transitoire responsable d'un bourgeonnement d'où sont émises des microparticules porteuses également de phosphatidylsérine et d'antigènes (en bleu) pouvant être caractéristiques de la cellule d'origine. Seul le transport unidirectionnel est représenté, en raison du manque de données concernant la fonction exacte des scramblases [22]. La nature du transporteur des aminophospholipides (en rose) reste à déterminer. Une fois la phosphatidylsérine accessible, les complexes enzymatiques de la coagulation peuvent se former. Ainsi l'enzyme $(\varepsilon)$ et le substrat (S), dépendants de la vitamine $K$, interagissent avec la phosphatidylsérine exposée par l'intermédiaire du calcium $\left(\mathrm{Ca}^{2+}\right)$. Le cofacteur (C) s'ancre dans la membrane de façon indépendante du calcium, mais toujours dépendante de la présence de la phosphatidylsérine. À partir de son substrat spécifique, chaque complexe produit l'enzyme suivante de la cascade de coagulation. Le dernier complexe, prothrombinase représenté ici, peut être reproduit in vitro pour détecter fonctionnellement l'exposition de phosphatidylsérine à la surface cellulaire. En effet, la quantité de thrombine produite à partir du substrat prothrombine (S) par l'enzyme facteur $X$ activé $(\varepsilon)$ en présence de cofacteur $V$ activé $(C)$ est proportionnelle au degré d'expression de la phosphatidylsérine. C. Dans le syndrome de Scott, la phosphatidylsérine n'est pas exposée à la surface des cellules soumises à une stimulation procoagulante, d'où l'absence d'assemblage des complexes enzymatiques de la coagulation et le phénotype hémorragique associé. 
après exposition de $P S$ et $P \varepsilon$, provoque une surcharge transitoire de PL dans le feuillet externe. Ce déséquilibre crée une augmentation de la courbure de la membrane qui forme des «bourgeons» à partir desquels les MP sont libérées [5]. L'exposition de la PS et l'émission de MP sont des événements quasi concomitants et associés [1] même si l'exposition de la PS semble être un prérequis à la formation de MP [4].

\section{Apoptose}

La mort cellulaire par apoptose est essentielle au maintien de l'homéostasie dans les organismes multicellulaires. Elle permet l'élimination de cellules indésirables sans provoquer de réaction inflammatoire et tient une place primordiale dans des processus normaux comme l'embryogenèse ou l'élimination des cellules immunitaires auto-réactives ${ }^{1}$. À l'inverse, une apoptose excessive accompagne des maladies comme le sida, l'athérosclérose ou certaines maladies dégénératives.

Concernant les répercussions membranaires de l'apoptose, l'exposition de la PS à la surface cellulaire est considérée comme un événement précoce, suivi d'une libération dans le

$(\rightarrow) \mathrm{m} / \mathrm{s}$ $2001, n^{\circ} 3$, p. 385 milieu extracellulaire des MP porteuses de PS et des marqueurs protéiques spécifiques de la cellule d'origine. L'exposition de PS est une caractéristique géné-

rale des cellules apoptotiques indépendamment du stimulus apoptogène [6], et constitue l'un des déterminants de leur reconnaissance et de leur élimination $(\rightarrow)$.

\section{Réponse hémostatique}

Après l'activation cellulaire, la PS exposée recrute et concentre les facteurs de la coagulation à la surface membranaire (Figure 1).

Le caractère essentiel de la PS dans la coagulation est illustré par le syndrome de Scott. II s'agit d'une maladie hémorragique héréditaire rare caractérisée par des épisodes de saignements dus à un déficit d'exposition de la PS. Ce défaut empêche l'assemblage des complexes enzymatiques de la coagulation et résulte donc en un déficit de production de thrombine. L'émission de MP procoagulantes est quasi nulle contribuant à la tendance hémorragique. Étant donné que le phénotype Scott est retrouvé dans plusieurs cellules sanguines, il est légitime de penser que l'atteinte provient d'une cel-

\footnotetext{
${ }^{1}$ Cellules qui participent à la réponse immunitaire et qui sont capables de s'autoactiver. II s'agit de lymphocytes (B ou T) normalement présents, mais qui, à la suite d'une activation/régulation anormale, peuvent développer des désordres auto-
} immuns. lule souche hématopoïétique, participant à la formation des cellules sanguines [7].

\section{Implication de la PS dans la phagocytose}

L'élimination des cellules apoptotiques est très efficace, les rendant pratiquement indétectables in vivo. Récemment, des auteurs ont identifié un gène qui coderait pour un récepteur de la PS exposée à la surface des cellules apoptotiques [8], alors que d'autres ont proposé la glycoprotéine MFG-ع8 (milk fat globuleEGF factor 8)/lactadhérine comme facteur se liant spécifiquement à ces cellules par reconnaissance de la PS exposée [9].

\section{Régulation de l'exposition de PS}

\section{Les transporteurs de PL}

Le maintien de l'asymétrie membranaire suppose que, dans la cellule au repos, le flux net d'aminoPL vers le feuillet interne, rapatriant la PS ou la PE exposées [1], est assuré par un transporteur membranaire appelé flippase ou aminoPL translocase.

Dans les cellules stimulées, l'augmentation du $\mathrm{Ca}^{2+}$ cytosolique inhibe la flippase [1]. Or, cette inhibition ne suffit pas à assurer l'exposition rapide de la PS, et donc un transport vers le feuillet externe doit s'activer. II y aurait un «brassage» des PL (scrambling) vers les deux feuillets de la membrane plasmique assuré par une scramblase. De plus, un flux de PS vers le feuillet externe est établi par un transporteur spécifique encore méconnu.

\section{Scramblase}

Une scramblase purifiée, reconstituée dans des liposomes, permet le transport bidirectionnel et aspécifique de PL entre les deux feuillets de la membrane [10]. Cette activité nécessite une concentration élevée de $\mathrm{Ca}^{2+}$ mais pas d'ATP. Des études réalisées chez des souris dont le gène de la scramblase a été invalidé montrent que cette «activité scramblase», ubiquitaire, participe à des processus membranaires jouant un rôle dans l'hématopoïèse, mais pas dans la réponse procoagulante [11].

\section{Floppase}

Le mouvement vectoriel (unidirectionnel) spécifique de PS pourrait être catalysé par une floppase, dépendante de I'ATP et du $\mathrm{Ca}^{2+}$ qui agirait en sens inverse de la flippase [12]. Dans les plaquettes, la floppase aurait une capacité de transport entre les deux feuillets de la membrane plasmique 20 fois supérieure à celle de la floppase du globule rouge, lui permettant ainsi d'assurer ses fonctions hémostatiques spécifiques. 


\section{Transporteurs ABC (ATP-binding cassette)}

Chez l'homme, les multidrug-resistant $P$-glycoproteins sont connues pour le rôle qu'elles jouent dans l'élimination des xénobiotiques. Un transporteur de cette famille externaliserait spécifiquement la PC, ce qui renforcerait I'hypothèse de transporteurs spécifiques de la PS.

Les résultats obtenus avec des souris dont les gènes $A B C A 4$ ou $A B C A 1$ ont été invalidés suggèrent qu'ABCA4 agirait comme un transporteur de $P E$ [13] alors qu'ABCAl participerait à l'exposition de PS et à l'efflux de cholestérol [14]. Les souris $A B C A 1^{-1-}$ présentent un phénotype rappelant celui des patients atteints d'un syndrome de Scott associant hémorragies et déficit d'exposition de PS.

\section{Le PIP}

Certains auteurs [15] ont proposé que le phosphatidylinositol-4, 5 biphosphate $\left(\mathrm{PIP}_{2}\right)$ jouerait un rôle dans la perte de l'asymétrie membranaire. L'interaction du complexe $\mathrm{PIP}_{2}-\mathrm{Ca}^{2+}$ pourrait former des microdomaines qui déstabiliseraient la bicouche lipidique et permettraient l'échange PS/PC. Or, ce complexe serait incapable d'induire une redistribution des PL membranaires des érythrocytes dans le syndrome de Scott [16], suggérant l'implication d'autres mécanismes dans l'exposition de la PS. Le $\mathrm{PIP}_{2}$ serait un régulateur positif du scrambling induit par le $\mathrm{Ca}^{2+}$ car les agents qui favorisent la formation de domaines $\mathrm{PIP}_{2}$ provoquent une redistribution des PL membranaires [15].
Rôle du $\mathrm{Ca}^{2+}$, des rafts et du cytosquelette

Dans les cellules activées, une augmentation soutenue de $\left[\mathrm{Ca}^{2+}\right]_{i}$ est nécessaire à l'externalisation de PS et à l'émission de MP. Dans des cellules issues d'une patiente atteinte du syndrome de Scott, une entrée insuffisante de $\mathrm{Ca}^{2+}$ à travers la membrane plasmique (entrée capacitative de $\left.\mathrm{Ca}^{2+}[\varepsilon C C]\right)^{1}$ a été observée et serait associée au défaut d'exposition de PS [17], la scramblase restant fonctionnelle [18].

Dans les cellules mégacaryocytaires de la lignée HEL, lorsque l'ECC est inhibée, l'exposition de la PS est aussi réduite [19]. Les protéines TRP (transient receptor potential) chez Drosophila melanogaster et leurs homologues chez les mammifères (TRPC) sont les canaux potentiels responsables de l' $E C C$. Nous avons observé que l'influx calcique à travers le canal TRPCI peut régler le degré d'exposition de la PS [20].

En outre, l'intégrité des rafts est essentielle pour une $\varepsilon C C$ et une exposition de la PS normales. Après stimulation, la PS exposée est co-localisée avec les rafts (Figure 2), et l'externalisation de la PS est inhibée lorsqu'on perturbe l'organisation des rafts, suggérant que les transporteurs ou les éléments régulateurs de ce mécanisme sont recrutés soit dans les rafts, soit à proximité [20].

Le cytosquelette est impliqué dans l'exposition de la PS et la formation de MP [5]. Dans les cellules mégacaryo-

${ }^{1}$ Une entrée capacitative de $\mathrm{Ca}^{2+}$ correspond à l'entrée de $\mathrm{Ca}^{2+}$ qui peut recharger les stocks du réticulum endoplasmique préalablement vidés.

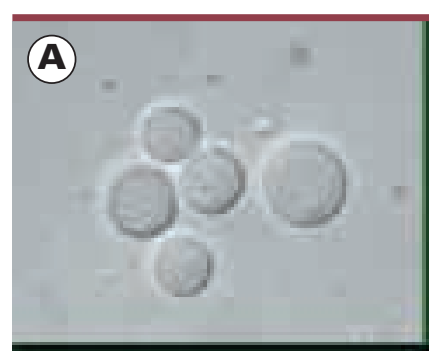

CTX-FITC

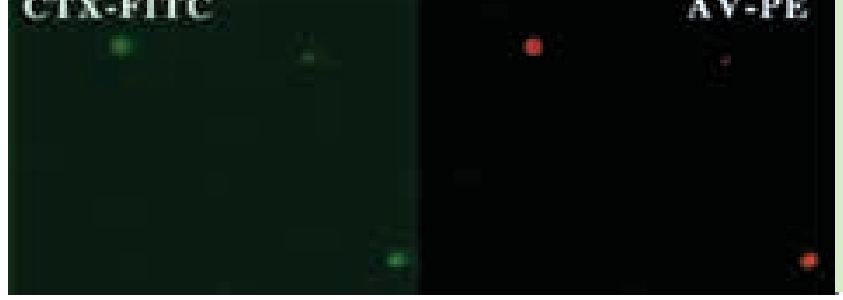

\section{Superposition} e
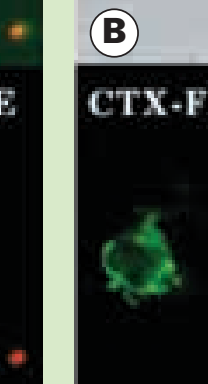
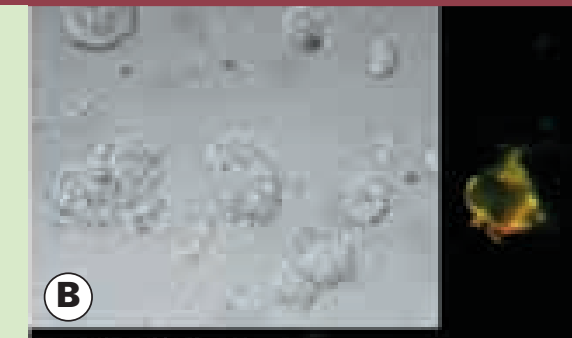

Superposition

Figure 2. Détection par immunofluorescence de la co-localisation de la phosphatidylsérine exposée et des rafts membranaires dans les cellules mégacaryocytaires HEL avant ( $A$ ) et après (B) stimulation par le ionophore calcique A23187. La phosphatidylsérine est marquée par l'annexine Vphycoérythrine ( $A V-P E$, en rouge) et les rafts par la toxine du choléra-fluorescéine isothiocyanate (CTX-FITC, en vert). La toxine du choléra marque le ganglioside GMl, un constituant habituel des rafts. La superposition des deux images démontre une telle co-localisation. 
cytaires HEL, l'externalisation de la PS serait réglée par la polymérisation du cytosquelette d'actine et la voie des MAP-kinases [19, 20]. Les mécanismes supposés participer à la régulation de l'externalisation de PS sont schématisés dans la Figure 3.

\section{Externalisation de la PS}

dans la réponse hémostatique versus apoptose

L'exposition de la PS est un élément déterminant pour la reconnaissance et l'élimination des cellules apoptotiques par les phagocytes. Le déficit génétique d'exposition de PS dans les cellules qui proviennent de patients atteints de syndrome de Scott, fait de celles-ci un excellent modèle pour étudier ce processus dans l'apoptose. Les cellules apoptotiques provenant de patients atteints du syndrome de Scott - notamment des lymphocytes B transformés par l'EBV - sont capables d'externaliser la PS [21]. Ces résultats suggèrent que l'exposition de PS, dans les réactions de l'hé-

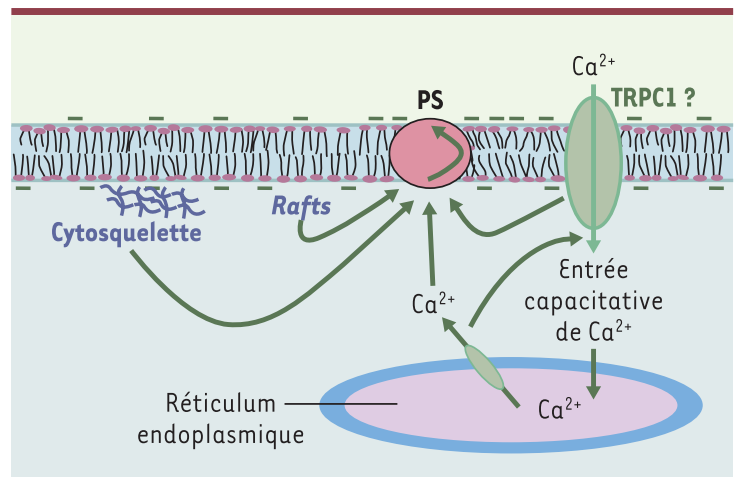

Figure 3. Mécanismes intracellulaires qui règlent l'externalisation de la phosphatidylsérine. Après une stimulation, le calcium $\left(\mathrm{Ca}^{2+}\right)$ est libéré à partir des réservoirs intracellulaires. La déplétion de ces stocks conduit à l'activation de canaux calciques (en vert) localisés dans la membrane plasmique et à un influx de calcium, aussi appelé entrée capacitative de calcium qui sert, en partie, à augmenter $\left[\mathrm{Ca}^{2+}\right]_{i}$ et à reconstituer les stocks du réticulum endoplasmique préalablement vidés. La nature des canaux qui participent à l'entrée capacitative de calcium reste à déterminer. Ce rôle pourrait être joué par les protéines dites TRP (transient receptor potential) chez Drosophila melanogaster et quelques homologues chez les mammifères (TRPC). L'entrée capacitative de calcium doit être normale pour permettre une externalisation normale de la phosphatidylsérine comme cela a été mis en évidence grâce aux inhibiteurs de cette voie d'entrée de calcium. L'intégrité de microdomaines lipidiques de type rafts et celle du cytosquelette sont nécessaires pour une exposition normale de la phosphatidylsérine. L'intégrité des rafts est essentielle pour une entrée capacitative normale de calcium. mostase et l'apoptose, pourrait être réglée par des mécanismes différents.

\section{Signification physiopathologique des MP}

La vésiculation membranaire serait également une caractéristique générale de la cellule apoptotique, une corrélation ayant été montrée entre le degré d'apoptose et le degré de vésiculation [22]. Les MP produites pendant l'apoptose, ou après stimulation cellulaire, ont une durée de vie plus longue que la durée de vie des cellules émettrices, probablement en raison de leur petite taille, inadaptée à la phagocytose, et de leur aptitude à diffuser, d'où l'intérêt des MP pour la détection d'une activation cellulaire in vivo.

\section{Les MP, marqueurs de l'activation cellulaire in vivo} Dans l'hémoglobinurie paroxystique nocturne, les MP circulantes, essentiellement plaquettaires, sont porteuses d'un potentiel procoagulant [23]. Les MP de la plaque d'athérome, porteuses de PS, peuvent catalyser les réactions procoagulantes [24]. Des taux élevés de MP d'origine endothéliale ont été observés chez les patients atteints de syndrome coronarien aigu [25]. Le taux élevé de MP circulantes, chez des patientes ayant des avortements spontanés à répétition, a été proposé comme un marqueur d'activation cellulaire reflétant de possibles complications thrombotiques (coagulation intravasculaire) et/ou des troubles du développement fœtal [26].

Des taux élevés de MP CD4 ${ }^{+}$ont été détectés chez des patients infectés par le $\mathrm{VIH}$, et pourraient être le témoin de la disparition des cellules T CD4 ${ }^{+}$dont l'apoptose reste très difficile à détecter in vivo [22]. Ces exemples montrent l'intérêt des MP pour le diagnostic de maladies associées à une stimulation ou à une mort cellulaires excessives. De plus, la régulation de la production des MP devrait permettre le suivi du traitement dans ces maladies.

\section{Les MP, acteurs de l'inflammation} et de la stimulation cellulaire

N. Satta et al. [27] ont montré que les MP provenant de monocytes stimulés par des agents infectieux disséminent une activité procoagulante double (PS et facteur tissulaire). De même, les MP circulantes d'origine leucocytaire $^{1}$ sont capables d'induire une réponse inflammatoire [28].

${ }^{1}$ Dans l'étude réalisée par N. Satta et al. [27], les MP sont produites par des monocytes isolés et purifiés. En revanche, les MP utilisés dans l'étude de M. Mesri et D.C. Altieri [28] sont d'origine leucocytaire sans précision du sous-type de cellule. 
Les MP, selon leur taux et leurs cellules d'origine, peuvent ainsi moduler les fonctions de cellules avec lesquelles elles interagissent, et parfois avec des conséquences délétères. Ainsi, après activation plaquettaire, les MP deviennent des agonistes grâce aux messagers lipidiques qu'elles transportent [29]. Les MP porteuses du ligand apoptogène FasL, parfois issues de cellules tumorales, sont susceptibles d'induire l'apoptose des cellules $T$, constituant un mécanisme d'échappement anti-tumoral [30]. De plus, les MP circulantes chez les patients ayant un infarctus du myocarde seraient responsables d'altérations de fonctions endothéliales [31]. Ces observations démontrent le bénéfice que l'on pourrait tirer d'une possible modulation du taux de MP en agissant sur l'externalisation de PS.

\section{Conclusions et perspectives}

Une meilleure connaissance des mécanismes régulateurs des mouvements transmembranaires de PL devrait permettre de moduler leur potentiel pro-inflammatoire, apoptogène, auto-immun ou pro-thrombotique, en agissant sur les degrés d'exposition de la PS et d'émission de MP procoagulantes.

Une approche pharmacologique réduisant le taux de MP semble pertinente vis-à-vis de cellules spécialisées comme les plaquettes. Cependant, il ne faut pas ignorer que les MP peuvent être «interprétées» dans d'autres processus, physiologiques cette fois, comme vecteurs transcellulaires de messages biologiques bénéfiques. Cet axe a été peu exploré jusqu'ici, mais on ne pourra envisager de moduler sélectivement les degrés d'externalisation de PS et de vésiculation membranaire dans certains types cellulaires, que si les fonctions de base dans d'autres cellules ou tissus n'en sont pas affectées. $\diamond$

\section{REMERCIEMENTS}

Nous exprimons nos remerciements les plus sincères aux membres de notre équipe, B. Hugel, D. Kerbiriou-Nabias et F. Toti pour leur aide constante dans la réalisation de ce projet de recherche, ainsi qu'à D. Meyer, Directrice de I'U.143 INSERM, pour son soutien permanent.

\section{SUMMARY}

Plasma membrane remodelling and cell stimulation For a long time the plasma membrane has been considered as a simple barrier between the extracellular and intracellular milieu. Now, it is well accepted that it plays a pivotal role in many physiological processes allowing the communication of cells with their environment. On the one hand, the plasma membrane directly participates in intracellular signaling, on the other hand, changes in membrane structure contribute to the transcellular transfer of biological information. This review analyses the most recent features concerning the plasma membrane plasticity, with a special focus on the intracellular signaling pathways involved in the regulation of the loss of membrane phospholipid asymmetry during cell activation. The pathophysiologic consequences of microparticle/microvesicle shedding from membrane blebs are briefly exposed. $\diamond$

\section{RÉFÉRENCES}

1. Zwaal RF, Schroit AJ. Pathophysiologic implications of membrane phospholipid asymmetry in blood cells. Blood 1997; 89: 1121-32.

2. Simons K, Toomre D. Lipid rafts and signal transduction. Nature Rev Molecular Cell Biol 2000; 1 : 31-9.

3. Edidin M. Shrinking patches and slippery rafts: scales of domains in the plasma membrane. Trends Cell Biol 2001; 11: 492-6.

4. Bassé $F$, Gaffet $P$, Rendu F, Bienvenüe A. Translocation of spin-labeled phospholipids through plasma membrane during thrombin-and ionophore A23187-induced platelet activation. Biochemistry 1993; 32 : 2337-44

5. Fox JE, Austin CD, Boyles JK, Steffen PK. Role of the membrane skeleton in preventing the shedding of procoagulant-rich microvesicles from the platelet plasma membrane. J Cell Biol 1990; 111 : 483-93.
6. Martin SJ, Reutelingsperger (PM, McGahon AJ, et al. Early redistribution of plasma membrane phosphatidylserine is a general feature of apoptosis regardless of the initiating stimulus: inhibition by overexpression of $\mathrm{Bcl}-2$ and Abl. J Exp Med 1995; 182: 1545-56.

7. Toti F, Satta N, Fressinaud $\varepsilon$, Meyer D, Freyssinet JM. Scott syndrome characterized by impaired transmembrane migration of procoagulant phosphatidylserine and hemorrhagic complications, is an inherited disorder. Blood 1996; 87: 1409-15.

8. Fadok VA, Bratton DL, Rose $D M$, et al. A receptor for phosphatidylserinespecific clearance of apoptotic cells. Nature 2000 ; 405: 85-90. 
9. Hanayama R, Tanaka M, Miwa K, et al. Identification of a factor that links apoptotic cells to phagocytes. Nature 2002; 417: 182-7.

10. Zhou Q, Zhao J, Stout JG, Luhm RA, Wiedmer T, Sims PJ. Molecular cloning of human plasma membrane phospholipid scramblase. A protein mediating transbilayer movement of plasma membrane phospholipids. J Biol Chem 1997; 272: 18240-4.

11. Zhou Q, Zhao J, Wiedmer T, Sims PJ. Normal hemostasis but defective hematopoietic response to growth factors in mice deficient in phospholipid scramblase 1. Blood 2002; 99: 4030-8.

12. Gaffet $P$, Bettache $N$, Bienvenüe A. Transverse redistribution of phospholipids during human platelet activation: evidence for a vectorial outflux specific to aminophospholipids. Biochemistry 1995; 34 : 6762-9.

13. Weng J, Mata NL, Azarian SM, et al. Insights into the function of Rim protein in photoreceptors and etiology of Stargardt's disease from the phenotype in abcr knockout mice. Cell 1999; 98: 13-23.

14. Hamon Y, Broccardo $C$, Chambenoit 0 , et al. $A B C 1$ promotes engulfment of apoptotic cells and transbilayer redistribution of phosphatidylserine. Nat Cell Biol 2000; 2 : 399-406.

15. Bucki R, Giraud F, Sulpice JC. Phosphatidylinositol 4,5-bisphosphate domain inducers promote phospholipid transverse redistribution in biological membranes. Biochemistry 2000; 39 : 5838-44.
16. Bevers EM, Wiedmer T, Comfurius $P$, et al. The complex of phosphatidylinositol 4,5biphosphate and calcium ions is not responsible for $\mathrm{Ca}^{2+}$-induced loss of phospholipid asymmetry in the human erythrocyte: a study in Scott syndrome, a disorder of calciuminduced phospholipid scrambling. Blood 1995; 86: 1983-91.

17. Martínez MC, Martin S, Toti $F$, et al. Significance of capacitative $\mathrm{Ca}^{2+}$ entry in the regulation of phosphatidylserine expression at the surface of stimulated cells. Biochemistry 1999; 38: 10092-8.

18. Janel N, Leroy C, Laude I, et al. Assessment of the expression of candidate human plasma membrane phospholipid scramblase in Scott syndrome cells. Thromb Haemost 1999; 81 : 322-3.

19. Kunzelmann-Marche $C$, Freyssinet JM, Martínez MC. Regulation of phosphatidylserine transbilayer redistribution by store-operated $\mathrm{Ca}^{2+}$ entry: role of actin cytoskeleton. J Biol Chem 2001; 276: 5134-9.

20. Kunzelmann-Marche $C$, Freyssinet JM, Martínez MC. Loss of plasma membrane phospholipid asymmetry requires raft integrity. Role of transient receptor potential channels and ERK pathway. J Biol Chem 2002; 277: 19876-81.

21. Martínez MC, Freyssinet JM. Deciphering the plasma membrane hallmarks of apoptotic cells: phosphatidylserine transverse redistribution and calcium entry. BMC Cell Biol 2001; 2 : 20.

22. Aupeix K, Hugel B, Martin T, et al. The significance of shed membrane particles during programmed cell death in vitro and in vivo in HIV-1 infection. J Clin Invest 1997; 99: 1546-54.

23. Hugel B, Socié G, Vu T, et al. Elevated levels of circulating procoagulant microparticles in patients with paroxysmal nocturnal hemoglobinuria and aplastic anemia. Blood 1999; 93: 3451-6.

24. Mallat Z, Hugel B, Ohan J, et al. Shed membrane microparticles with procoagulant potential in human atherosclerotic plaques: a role for apoptosis in plaque thrombogenicity. Circulation 1999; 99 : 348-53.

25. Mallat Z, Benamer H, Hugel $B$, et al. Elevated levels of shed membrane microparticles with procoagulant potential in the peripheral circulating blood of patients with acute coronary syndromes. Circulation 2000; 101 : 84l-3.

26. Laude I, RongièresBertrand C, Boyer-Neumann $C$, et al. Circulating procoagulant microparticles in women with unexplained pregnancy loss: a new insight. Thromb Haemost 2001; 85: 18-21.
27. Satta N, Toti F, Feugeas 0, et al. Monocyte vesiculation: a mechanism for dissemination of membrane-associated procoagulant activities and adhesion molecules following stimulation by lipopolysaccharide. J Immunol 1994; 153: 3245-55.

28. Mesri M, Altieri DC. Leukocyte microparticles stimulate endothelial cell cytokine release and tissue factor induction in a JNKI signaling pathway. J Biol Chem 1999; 274 : 23111-8.

29. Barry OP, Pratico D, Savani RC, Fitzgerald GA. Modulation of monocyteendothelial cell interactions by platelet microparticles. J Clin Invest 1998; 102: 136-44.

30. Albanese J, Meterissian S, Kontogiannea $M$, et al. Biologically active Fas antigen and its cognate ligand are expressed on plasma membrane-derived extracellular vesicles. Blood 1998; 91 : 3862-74.

31. Boulanger CM, Scoazec A, Ebrahimian $\mathrm{T}$, et al. Circulating microparticles from patients with myocardial infarction cause endothelial dysfunction. Circulation 2001 ; 104 : 2649-52.

\section{TIRÉS À PART}

J.M. Freyssinet 